\title{
Nicht Fisch, nicht Fleisch, aber unverzichtbar
}

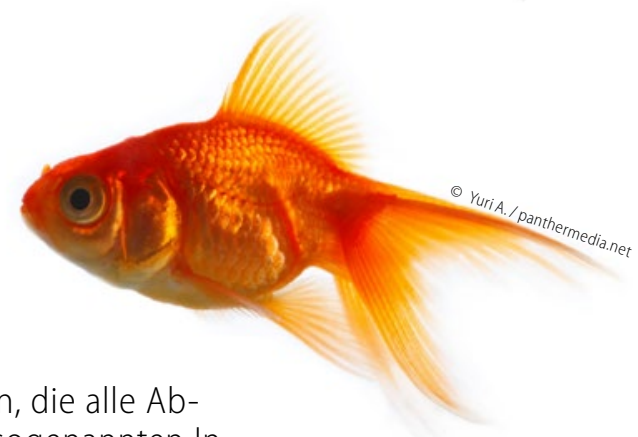

\begin{abstract}
Buchhaltung ist immer ein Abbild der Realität und muss daher Funktionen bieten, die alle Abläufe des betrieblichen Alltags darstellen können. Sehr hilfreich hierbei sind die sogenannten Interimskonten. Gablers Wirtschaftslexikon definiert diese als "Zwischenkonto; Hilfskonto, das lediglich Verrechnungsfunktionen erfüllt".
\end{abstract}

Wie der Name schon sagt, sind die Posten auf diesen Konten für eine zeitlich befristete Dauer dort verbucht. Es handelt sich um ergebnisneutrale Konten, die in der betriebswirtschaftlichen Auswertung weder als Aufwand noch als Erlös ersichtlich sind. Lediglich in der Liquiditätsbetrachtung werden die Interimskonten aufgeführt.

Um die auf dem Verrechnungskonto „geparkten“ Beträge in der Erfolgsrechnung wirksam zu machen, müssen diese den entsprechenden Kosten- oder Erlöskonten zugebucht und aus dem Verrechnungskonto ausgebucht werden.

Handelt es sich bei den gebuchten Beträgen um „durchlaufende Posten“, die den Unternehmenszweck gar nicht betreffen, müssen diese Beträge solange dort verbleiben, bis sie dem eigentlichen Empfänger ausgezahlt beziehungsweise vom Schuldner, für den der Betrag verauslagt wurde, erstattet wurden.

\section{Transparenz durch Nutzung von Verrechnungskonten} Jeder kennt aus dem Alltag Situationen, in denen man sich die Verrechnung von verauslagten oder geliehenen Beträgen notieren muss. Oder es wird Geld irgendwo entnommen, das an anderer Stelle wieder in das Unternehmen einfließen soll. In der Buchhaltung ist dies über Verrechnungskonten einfach zu lösen.

So dient beispielsweise das Verrechnungskonto Geldtransit der Kontrolle und Transparenz. Bei allen Bewegungen zwischen Finanzkonten innerhalb der Praxis (zum Beispiel Geldentnahme aus Kasse und Einzahlung auf Bankkonto oder Überweisung von Praxis-Bankkonto zu Praxis-Bankkonto) wird „Geldtransit“ als Gegenkonto angesprochen. Auf diesem Konto ist dann immer sehr gut zu ersehen, ob der Betrag auch in voller Höhe auf dem Zielkonto eingegangen ist. Also: Ist das aus der Kasse entnommene Geld auch auf dem Bankkonto eingezahlt worden? Hat die Überweisung von Bankkonto A auf Bankkonto B funktioniert?

Weiter eignen sich Verrechnungskonten auch für die vereinfachte Bearbeitung und Verbuchung von Kreditkartenabrechnungen. Es wird auf dem Bankkonto vom Karteninstitut lediglich ein pauschaler Betrag belastet, der sowohl Kartenzahlungen als auch eventuelle Gutschriften sowie Kosten kumuliert enthält. Verbucht man diesen Betrag bei Bearbeitung der Bankauszüge zunächst ungesplittet gegen ein Verrechnungskonto und bei Vorliegen der detaillierten Kreditkartenabrechnung diese ebenfalls dorthin, ergibt sich zum einen ein klares Bild der Bewegungen auf dem Kreditkartenkonto und außerdem jederzeit über den Saldo die Kontrolle der Abrechnung.

\section{Geeignet bei Praxisübernahme oder -abgabe}

Typische Anwendungen treten auch häufig im Zusammenhang mit Abgabe oder Übernahme der Zahnarztpraxis auf. In der Übergangsphase kommt es häufig zu Zahlungen von Eingängen auf (alte) Bankkonten, die dann mit der Buchhaltung der neuen Praxis zu verrechnen sind. Als Gegenkonto ist hier ein Verrechnungskonto geeignet. Sobald der verauslagte Betrag eingegangen oder die fälschlich erhaltene Patientenzahlung an die neue Praxis weitergeleitet wurde und auf dem Verrechnungskonto gegengebucht ist, ist dieses ausgeglichen.

Allen Interims- beziehungsweise Verrechnungskonten gemeinsam ist jedoch, dass sie am Ende des Jahres einen Null-Saldo aufweisen sollen. Ist das nicht der Fall, muss das Konto geklärt oder vorgetragen werden. Möglich ist etwa, dass noch ein Betrag ausstehend ist oder eine Gegenbuchung nicht korrekt durchgeführt wurde.

Unser Tipp: Fibu-doc-Anwender erhalten umfangreichen Support über die Hotline, auch bei der Anleitung zur Saldenabstimmung!

Das in den Artikeln dargestellte Buchhaltungskonzept basiert auf der zahnarztspezifischen Buchhaltungssoftware fibu-doc und wird vom FVDZ unterstützt.
Seminare:
Personalverwaltung
Planen - Steuern - Analysieren 05.04.2017 Nürnberg
Personalverwaltung
Planen - Steuern - Analysieren $\quad$ 19.04.2017 Kassel
Praxisbuchhaltung
Leicht und verständlich
21.06.2017 Würzburg 